\title{
Observational Study of the Effect of Moderate and High-Intensity Exercise on Insomnia
}

\author{
David Redding, DOㅜ; Angela Maron, OMS'; Emily Venvertloh, OMS ${ }^{1}$; \\ Tania C. Ghazarian, $\mathrm{OMS}^{1}$; Erika Polanco, $\mathrm{MS}^{2}$ \\ ${ }^{1}$ College of Osteopathic Medicine of the Pacific, Western University of Health Sciences, Pomona, CA \\ ${ }^{2}$ School of Medicine, Wayne State University, Detroit, MI
}

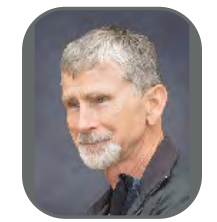

David Redding, D0

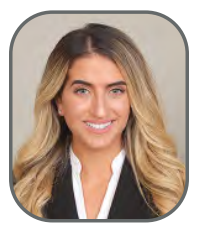

Angela Maron, OMS

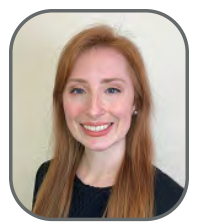

Emily Venvertloh, OMS

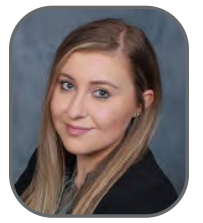

Tania C. Ghazarian, OMS

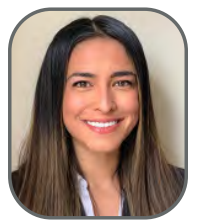

Erika Polanco, MS

\section{CORRESPONDENCE:}

David Redding, DO

redding@westernu.edu

DOI: $10.38206 / 140107$

KEYWORDS:

Exercise; High-Intensity; Insomnia; Insomniac; Moderate-intensity; Sleep latency;

Sleep Quality

\section{ABSTRACT}

The purpose of this observational study is to determine whether moderate-intensity exercises improve the quality of sleep for subjects with insomnia. The duration of this observational study was four weeks. For the first two weeks of the observational study, subjects served as their own control and completed a sleep log. At the beginning of the third week, subjects were randomly split into (1) Group One with moderate-intensity exercise or (2) Group Two with high-intensity exercise. Two-tailed t-tests displayed significant improvements in quality of sleep with the implementation of both moderate and high-intensity exercise. However, Group One participants were found to feel significantly more well-rested than Group Two. The moderate-intensity exercise group showed improvement in quality of sleep, the number of awakenings, and restfulness. In comparison, the high-intensity exercise group demonstrated improvement in the number of awakenings and daytime sleepiness; although, without reported improvement in sleep quality. There were variables in participants' sleeping environments, including changes in sleep location, travel, sleep-inducing medication, alcohol use, anxiety, unanticipated noise disturbances, and use of electronic devices before bed that was not controlled for in this observational study.

SUMMARY:

\section{Current Knowledge/Observational Study Rationale:}

Exercise has been proposed as an alternative treatment for insomnia; however, there has been a limited exploration into the recommended exercise modality. This observational study was performed to assess which exercise regimen, moderate or high-intensity, is most effective in treating patients with insomnia.

\section{Study Impact:}

This is one of the first observational studies showing that both moderate and high-intensity exercise can improve different attributes of insomnia. This observational study paves the way for future research exploring the effects of exercise on patients with insomnia within their own home environment utilizing larger sample sizes. 


\section{INTRODUCTION}

Insomnia is a sleeping disorder characterized by the inability to fall or stay asleep. ${ }^{1}$ It has a prevalence of approximately 6-10\% of the general public, with 33\% reporting some form of insomnia symptoms. ${ }^{2}$ DSM V describes Insomnia Disorder as dissatisfaction with sleep associated with at least one of the following: difficulty initiating sleep, maintaining sleep with frequent awakenings, and/or returning to sleep in the early morning. ${ }^{2}$ Consequences of sleep disruption include an increased stress response, somatic pain, reduced quality of life, emotional distress, mood disorders, and memory deficits. ${ }^{1}$ Long term consequences of insomnia can lead to chronic disease. ${ }^{1}$ Current approach to managing insomnia includes prescription medications and over-the-counter supplements that may be costly and associated with adverse side effects.

Previous studies have found an association between moderate-intensity exercises and reduced sleep latency as well as anxiety levels. ${ }^{3}$ In these studies, participants were analyzed in a laboratory setting that may not reflect the participants' natural sleep environment. This questions whether the same results may be reproduced if participants implemented these exercises at home. Furthermore, minimal studies have incorporated daily sleep logs to account for day-to-day sleep quality and confounding variables. The purpose of this observational study is to assess whether exercise can be utilized as an effective means of improving symptoms of insomnia in a home setting. This observational study compares two types of exercise routines to explore which is more effective in inducing sleep: high-intensity or moderateintensity. The moderate-intensity protocol incorporates slow, deep breathing and stretching positions that increase blood oxygenation and body awareness. ${ }^{4}$ The high-intensity exercise protocol includes jogging in place, alternating cross-punching and kicking, jumping jacks, and "air" jump rope. The objective of this observational study was to analyze the effect of two types of physical exercise on the subjective sleep quality of participants with self-reported insomnia.

\section{METHODS}

An informative flyer was sent out to all students, staff, and faculty at Western University of the Health Sciences, inviting them to participate in this observational study if they have been experiencing insomnia. Inclusion criteria included self-reported insomnia and age between 18-60 years old. Exclusion criteria included pregnancy, those with difficulty balancing, and/or inability to perform exercises.

A total of 87 volunteers initially responded to participate in the observational study. Of these participants, four were excluded for being above the age of 60 , while only one was excluded for being pregnant. At the start of the observational study, 24 volunteers completed the enrollment process. They were randomly allocated using a number generator that assigned participants to either Group One with moderateintensity exercise or Group Two with high-intensity exercise. Most participants withdrew from the observational study by not completing the daily sleep log for the entire 28 days. By the end of the observational study, seven participants remained in Group One, while five remained in Group Two.

The length of the observational study was four weeks. The first two weeks was a control period where each participant continued their daily activities while completing a daily sleep log. The following two weeks were the experimental period where participants completed their assigned exercise regime and a daily sleep log. The sleep log was used to analyze the subjects' overall sleep quality and included questions assessing sleep latency, duration of sleep, number of awakenings throughout the night, amount of time needed to fall back asleep upon awakening, rating of restfulness felt in the morning, daytime sleepiness, and any confounding variables.

Group One was assigned a set of moderate-intensity stretching exercises that are believed to increase parasympathetic tone and decrease sympathetic activity during the postexercise recovery period. ${ }^{5}$ These exercises included head and neck stretching, trunk stretching, toe and heel rocking, alternate raising and stooping, and hip circles. Subjects were taught how to perform these exercises at the first meeting.

Group Two was assigned high-intensity exercises proposed to increase sympathetic activation. ${ }^{5}$ The high-intensity exercise protocol includes jogging in place, alternating cross-punching and kicking, jumping jacks, and "air" jump rope. These exercises were taught during the first meeting.

The exercise regimen for each group was approximately 10-15 minutes. The participants were instructed to perform the exercises each evening, 30 minutes before going to sleep. Each morning, the participants recorded their sleep quality 
on the designated log. After the four weeks, each group was assessed to determine if the assigned exercises impacted reported sleep quality. This was achieved using the first two weeks as control and the subsequent two weeks as the experimental period. The two groups were then compared to identify whether there was a significant difference in reported sleep quality. Results were calculated using paired two-tailed t-tests to compare the control period to the respective exercise periods.

\section{RESULTS}

The incorporation of moderate-intensity exercises (Group One) significantly improved self-reported overall quality of sleep compared to the control period (Figure 1, p-value of 0.000459); however, those who performed high-intensity exercises (Group Two) did not display significantly improved self-reported quality of sleep compared to their control period ( $p$-value 0.9). In respect to its control period, the reported duration of sleep in both the high-intensity and moderate-intensity exercise group was not significantly improved ( $p$-value of 0.095 and 0.527 , respectively). There were no significant changes in sleep latency in both the high-intensity and moderate-intensity exercise groups compared to their control periods ( $p$-value of 0.071 and 0.260 , respectively). The number of awakenings throughout the night significantly decreased in both the moderateintensity and high-intensity exercise groups compared to the respective control periods (Figure 2, p-value 0.004 and Figure 3, 0.03 respectively). Daytime sleepiness significantly decreased in the high-intensity group compared to the control period (Figure 4, p-value of 0.028); however, there was no significant difference in daytime sleepiness in the moderate-intensity group compared to the control ( $p$-value of 0.650). Moderate-intensity participants reported being significantly more rested in

\section{FIGURE 1.}

The average quality of sleep was reported on a scale of 1-10 for moderate-intensity exercise participants during control and exercise periods. A significant difference was observed between the control period and exercise periods ( ${ }^{*} p=.00046$, paired two-tailed t-test).

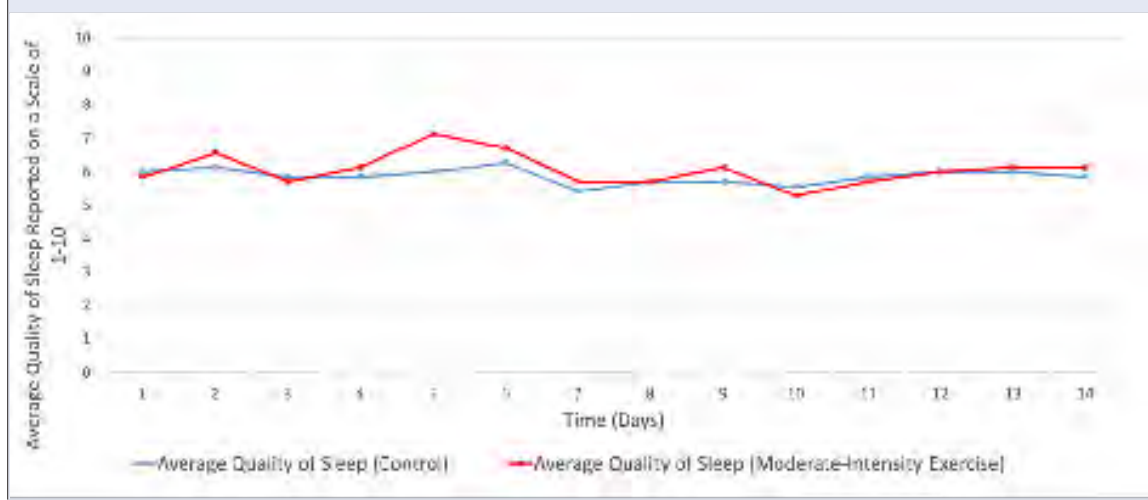

\section{FIGURE 2.}

Average amount of awakenings throughout the night for moderate-intensity exercise participants during control vs. exercise periods. A significant difference was observed between the control period vs. the exercise period ( ${ }^{\star} p=.00379$, paired two-tailed t-test).

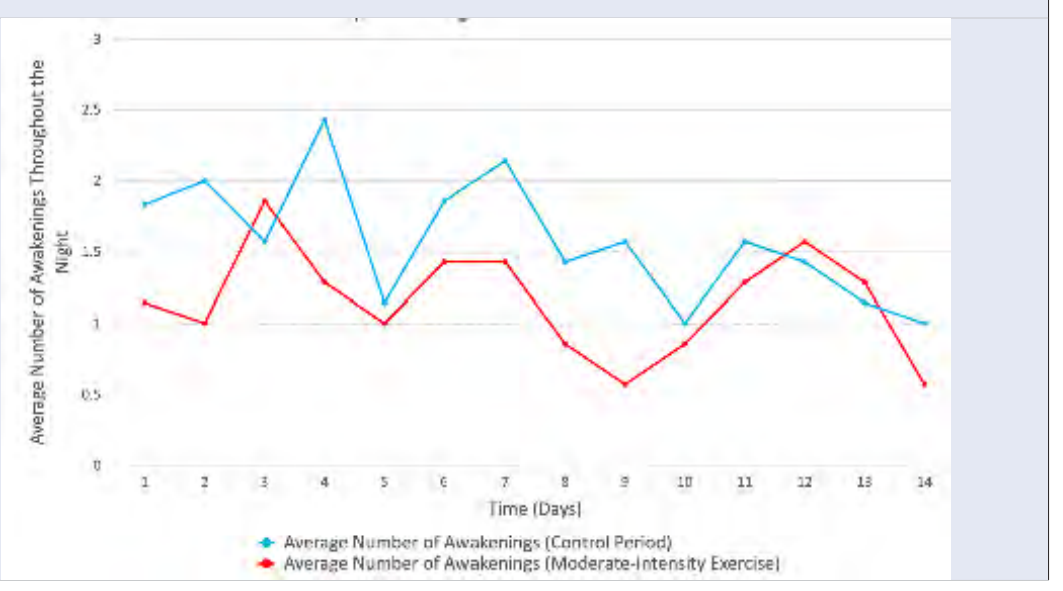

the exercise period compared to the control period (Figure 5, p-value 0.0002). However, there was no significant change in restfulness for the high-intensity participants compared to their control ( $p$-value 0.05508 ).

\section{DISCUSSION}

This observational study investigated the acute effect of moderate and high-intensity exercise on insomnia using different quantitative and qualitative parameters. Our original hypothesis was that exercise would alleviate self-reported insomnia, and more specifically, the moderateintensity exercise would be more effective at improving insomnia than highintensity exercise. Using ICSD-3's definition of insomnia, the observational study explored parameters around the time to fall asleep, times woken up in the middle of the night, restfulness, and daytime sleepiness. ${ }^{6}$ Other 
parameters not included in ICSD-3 but thought to be valuable to our observational study included the amount of time needed to fall back asleep upon awakening and any confounding variables.

After analyzing the effect of different exercise modalities, we observed that moderateintensity exercise significantly improved selfreported overall quality of sleep (Figure1) and restfulness (Figure 5). Increased parasympathetic activity has been shown to increase vagal tone, improving subjective sleep quality measures and anticipation of sleep onset.? Specifically, studies have shown that disorders that cause sleep disruption, i.e., insomnia, can lead to a reduction in parasympathetic tone and sympathovagal imbalance. ${ }^{8}$ This is one explanation for how the moderate-intensity exercise group had a significantly improved quality of sleep as opposed to the high-intensity exercise group. There have been studies that have identified that both stretching exercises, as seen in our moderate-intensity group, and HighIntensity Interval Training (HIIT) exercises can increase the parasympathetic tone during the post-exercise period; ${ }^{9}$ however, there is limited research on post-exercise parasympathetic effects in high-intensity exercise in the absence of interval training. Although some studies have concluded that any form of exercise can increase parasympathetic activity, specific studies should be conducted in the future addressing whether moderate-intensity exercise causes a greater increase in parasympathetic tone than highintensity exercise.

Of note, a significant decrease in the number of awakenings throughout the night was found for both the moderate and high-intensity groups (Figures 2 and 3). These results coincide, in part, with our original hypothesis that sleep would improve attributes of insomnia. This finding may be due to exercise increasing energy consumption and endorphin secretions, leading to a facilitation of sleep. ${ }^{10}$ This could indicate that any type of exercise could improve different variables of insomnia and is a possible avenue for future studies.

\section{FIGURE 3.}

Average number of awakenings throughout the night for high-intensity exercise participants during control vs. exercise periods. A significant difference was observed between the control period vs. the exercise period ( ${ }^{*} p=.0307$, paired two-tailed t-test).

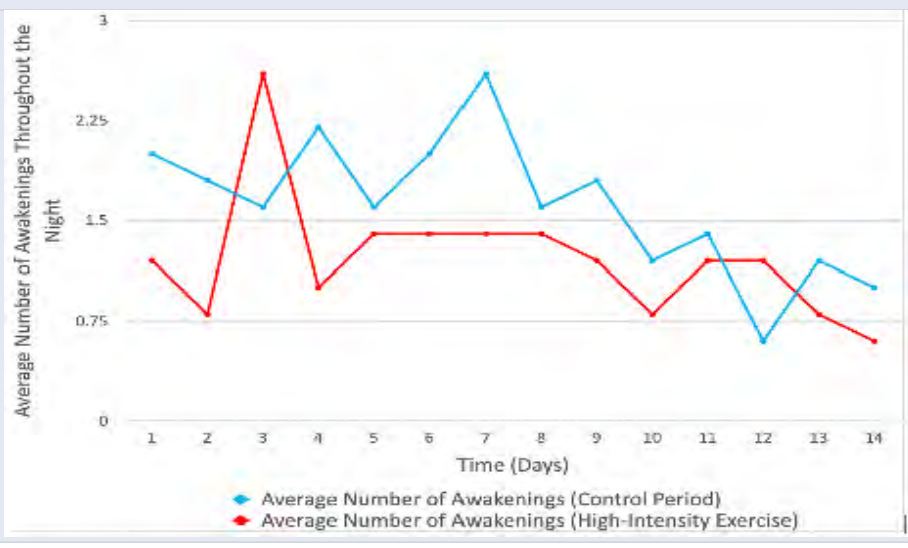

\section{FIGURE 4.}

Average rating of daytime sleepiness on a scale of 1-10 of high-intensity exercise participants during control period vs. exercise period. A significant difference was observed between the control period vs. the exercise period ( ${ }^{\star} p=.0284$, paired two-tailed $t$-test).

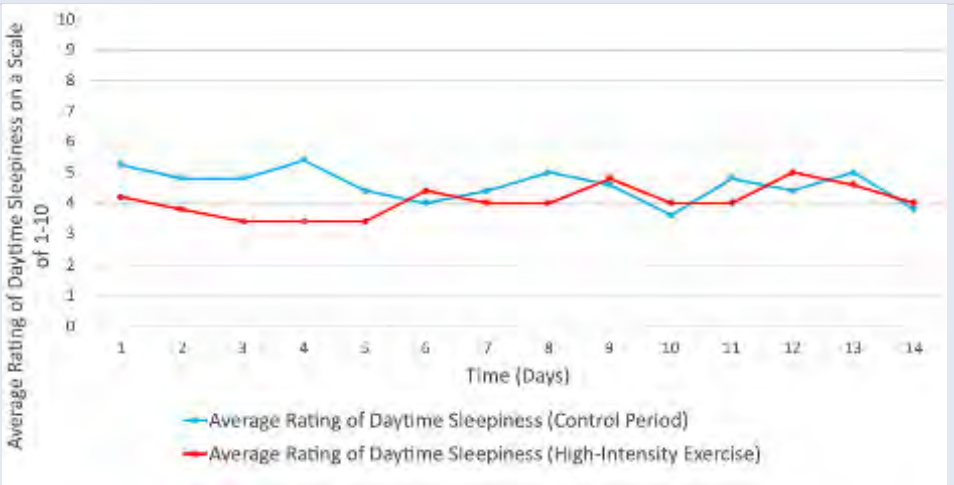

\section{FIGURE 5.}

Average rating of restfulness on a scale of 1-10 for moderate-intensity exercise during the control period vs. exercise period. A significant difference was observed between the control vs. exercise period ( ${ }^{*} p=.000186$, paired two-tailed t-test).

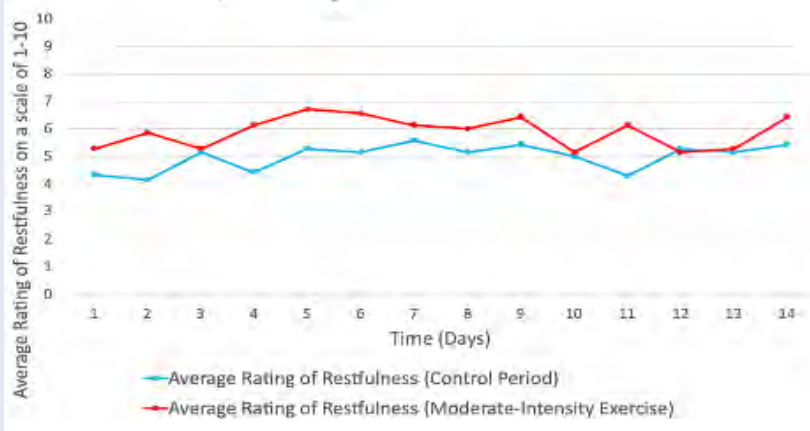


Interestingly, only the high-intensity group had a significant decrease in daytime sleepiness (Figure 4). This finding is the opposite of what was expected. It was originally hypothesized that the moderate-intensity exercise group would show an improvement in all variables of insomnia. This may be attributable to high-intensity exercise enhancing the post-exercise recovery period. ${ }^{9}$ Other variables found to not be significant in either group included sleep latency, amount of time to fall back asleep after awakening, and duration of sleep. These unanticipated results may be due to the small sample size, length of the observational study, and other confounding variables.

As part of the sleep log, patients also reported factors that they believe could have affected their sleep and confounded their results. During the control period of the moderateintensity group, participants noted possible disruptions to their sleep, including anxiety, neighbors fighting, hotel rooms, alcohol, colds, and camping. Participants noted basketball games before bed, late-night gaming, and disruption from pets in the moderate-intensity group during the exercise period. During the control period, high-intensity group participants noted early flights, wind, and a snoring partner. High-intensity group participants noted late-night studying, roommate's alarm clock, travel, and anxiety in the exercise period. These were extraneous variables that the participants noted as possible causes of sleep disturbances. The observational study attempted to mitigate confounding variables by implementing a control period, but there were inevitable changes to the participants' sleeping environments.

This observational study did have its limitations. The sample size began at $n=33$, but for various reasons, participants dropped out. If the observational study were to be replicated in the future, there would need to be an increase in sample size. Future studies should evaluate extraneous variables that may affect sleep quality, account for BMI, and only accept participants who will be in the same environment for the entire length of the observational study.

\section{AUTHOR DISCLOSURES:}

No relevant financial affiliations or conflicts of interest.

\section{REFERENCES}

1. Medic G, Wille M, Hemels ME. Short- and long-term health consequences of sleep disruption. Nat Sci Sleep. 2017; 9:151161. Published 2017 May 19. doi:10.2147/NSS.S134864

2. American Psychiatric Association. Diagnostic and Statistical Manual of Mental Disorders. 5th ed. Washington D.C.: 2013.

3. Passos GS, Poyares D, Santana MG, Garbuio SA, Tufik S, Mello MT. Effect of acute physical exercise on patients with chronic primary insomnia. J Clin Sleep Med. 2010;6(3):270-275.

4. Russo MA, Santarelli DM, O'Rourke D. The physiological effects of slow breathing in the healthy human. Breathe (Sheff). 2017;13(4):298-309. doi:10.1183/20734735.009817

5. Farinatti PT, Brandão C, Soares PP, Duarte AF. Acute effects of stretching exercise on the heart rate variability in subjects with low flexibility levels. J Strength Cond Res. 2011;25(6):15791585. doi:10.1519/JSC.0b013e3181e06ce1

6. The International Classification of Sleep Disorders. Darien, IL: American Academy of Sleep Medicine, 2014.

7. Werner GG, Ford BQ, Mauss IB, Schabus M, Blechert J, Wilhelm $\mathrm{FH}$. High cardiac vagal control is related to better subjective and objective sleep quality. Biol Psychol. 2015;106:7985. doi:10.1016/j.biopsycho.2015.02.004

8. Fink AM, Bronas UG, Calik MW. Autonomic regulation during sleep and wakefulness: a review with implications for defining the pathophysiology of neurological disorders. Clin Auton Res. 2018;28(6):509-518. doi:10.1007/s10286-018-0560-9

9. Besnier F, Labrunée M, Pathak $A$, et al. Exercise traininginduced modification in autonomic nervous system: An update for cardiac patients. Ann Phys Rehabil Med. 2017;60(1):2735. doi:10.1016/j.rehab.2016.07.002

10. Yang PY, Ho KH, Chen HC, Chien MY. Exercise training improves sleep quality in middle-aged and older adults with sleep problems: a systematic review. J Physiother. 2012;58(3):157-163. doi:10.1016/S1836-9553(12)70106-6 